\title{
LOG CONCAVE SEQUENCES OF SYMMETRIC FUNCTIONS AND ANALOGS OF THE JACOBI-TRUDI DETERMINANTS
}

\author{
BRUCE E. SAGAN
}

\begin{abstract}
We prove that various sequences of elementary and complete homogeneous symmetric functions are log concave or PF. As corollaries we show that certain sequences of $q$-binomial coefficients and $q$-Stirling numbers have these properties. The principal technique used is a combinatorial interpretation of determinants using lattice paths due to Gessel and Viennot [G-V 85].
\end{abstract}

\section{INTRODUCTION}

Let $\mathbf{Z}$ and $\mathbf{N}$ denote the integers and nonnegative integers respectively. Throughout this paper, indices for sequences and sums will range over $\mathbf{N}$. Also let $\mathbf{R}$ and $\mathbf{R}^{+}$stand for the reals and nonnegative reals.

A sequence of real numbers,

$$
\left(a_{k}\right)_{k \geq 0}=a_{0}, a_{1}, a_{2}, \ldots
$$

is log concave if

$$
a_{k-1} a_{k+1} \leq a_{k}^{2} \text { for all } k>0 .
$$

Log concave sequences arise in combinatorics, algebra, and geometry. Stanley offers a good survey of the subject in [Sta 89]. If the $a_{k}$ are all positive, then log concavity is equivalent to the seemingly stronger condition

$$
a_{k-1} a_{l+1} \leq a_{k} a_{l} \text { for all } 0<k \leq l .
$$

This, in turn, is clearly equivalent to the determinantal formula

$$
\left|\begin{array}{cc}
a_{l} & a_{l+1} \\
a_{k-1} & a_{k}
\end{array}\right| \geq 0 \text { for all } 0<k \leq l
$$

which is reminiscent of a $2 \times 2$ Jacobi-Trudi determinant (see Macdonald [Mad 79 , p. 25] or Sagan [Sag 90; Sag 91, pp. 154-159]).

Now let $q$ be an indeterminate. Recently there has been much interest in the $q$-analog of log concavity [But $90, \mathrm{Kra} 89$, Ler 90, Sag pr] as defined by Stanley

Received by the editors December 7, 1989.

1980 Mathematics Subject Classification (1985 Revision). Primary 05A15; Secondary 05A10, $11 \mathrm{~B} 65,11 \mathrm{~B} 73$.

Key words and phrases. Log concave, $q$-analog, $q$-binomial coefficients, $q$-Stirling numbers, symmetric function, Jacobi-Trudi determinants, Gessel-Viennot lattice paths.

Supported in part by NSF grant DMS 8805574. 
[private communication]. We must first define the $q$-analog of the relation $\leq$ which will be denoted $\leq_{q}$. Given two polynomials $f(q), g(q) \in \mathbf{R}[q]$, we write

$$
f(q) \leq_{q} g(q) \text { if and only if } g(q)-f(q) \in \mathbf{R}^{+}[q] \text {. }
$$

Equivalently, for every $k \geq 0$, the coefficient of $q^{k}$ in $f(q)$ is less than or equal to the corresponding coefficient in $g(q)$. This is a partial order on $\mathbf{R}[q]$. Now consider a sequence of polynomials in $\mathbf{R}[q]:\left(f_{k}(q)\right)_{k \geq 0}$. This sequence is said to be $q$-log concave if

$$
f_{k-1}(q) f_{k+1}(q) \leq_{q} f_{k}(q)^{2} \text { for all } k>0
$$

and it is strongly $q$-log concave if

$$
f_{k-1}(q) f_{l+1}(q) \leq_{q} f_{k}(q) f_{l}(q) \text { for all } 0<k \leq l .
$$

It is not hard to show that these two notions are not equivalent for general $q$ [Sag pr].

As examples, let $[n]=1+q+q^{2}+\cdots+q^{n-1}$ for $n \in \mathbf{N}$. Define the $q$ analogs of the binomial coefficients and the Stirling numbers of the first and second kinds by

$$
\begin{array}{rlrl}
{\left[\begin{array}{l}
n \\
k
\end{array}\right]} & =\left[\begin{array}{l}
n-1 \\
k-1
\end{array}\right]+q^{k}\left[\begin{array}{c}
n-1 \\
k
\end{array}\right] & & \text { for } n \geq 1, \text { with }\left[\begin{array}{l}
0 \\
k
\end{array}\right]=\delta_{0, k}, \\
c[n, k] & =c[n-1, k-1]+[n-1] c[n-1, k] & \text { for } n \geq 1, \text { with } c[0, k]=\delta_{0, k}, \\
S[n, k] & =S[n-1, k-1]+[k] S[n-1, k] & & \text { for } n \geq 1, \text { with } S[0, k]=\delta_{0, k},
\end{array}
$$

where $\delta_{0, k}$ is the Kronecker delta. These definitions are meant to apply for all $k \in \mathbf{Z}$ and $n \in \mathbf{N}$. The nonzero polynomials in each of these three families can be arranged in a triangle like Pascal's triangle for binomial coefficients. Considering the sequences formed by lines parallel to each side of each triangle, one can prove

Theorem 1.1. The following sequences are all strongly $q$-log concave,

1. for fixed $n:\left(\left[\begin{array}{l}n \\ k\end{array}\right)_{k \geq 0},(c[n, k])_{k \geq 0},(S[n, k])_{k \geq 0}\right.$,

2. for fixed $k:\left(\left[\begin{array}{l}n \\ k\end{array}\right]\right)_{n \geq 0},(S[n, k])_{n \geq 0}$,

3. for fixed $n, k:\left(\left[\begin{array}{c}n+j \\ k+j\end{array}\right]\right)_{j \geq 0},(c[n+j, k+j])_{j \geq 0},(S[n+j, k+j])_{j \geq 0}$.

All of the authors cited previously have contributed to proving parts of this theorem. (Butler and later Krattenhaler considered the $q$-binomial coefficients while Leroux and Sagan worked on the $q$-Stirling numbers.)

It is easy to see that the $q$-binomials and $q$-Stirlings are just specializations of elementary and complete symmetric functions. (We will make this explicit in §2.) As Stanton [private communication] pointed out, certain parts of the Theorem 1.1 now follow immediately from the nonnegativity of the coefficients of the Jacobi-Trudi determinants. In [Sag ta] we found other symmetric function identities that specialized to the rest of the cases above. Unfortunately, the principal technique used was induction, which is not very enlightening.

The purpose of the present work is to give combinatorial proofs of generalizations of these results. The crucial tool will be a method due to Gessel [Ges um] and Gessel-Viennot [G-V 85] for interpreting determinants as lattice paths. In the following section we will review some background material on symmetric functions. Next, the Gessel-Viennot approach will be outlined. Section 4 will 
contain the proofs of our main results, Theorems 4.1 and 4.3. In $\S 5$ we will consider the implications of these methods for PF (Pólya frequency) sequences. Our concluding section will contain some related results and open questions.

\section{SYMMETRIC FUNCTIONS}

Let $\mathbf{x}=\left\{x_{1}, x_{2}, \ldots\right\}$ be a countably infinite set of variables. Let $M$ be a multiset (= "set" with repetitions allowed) with elements from the positive integers. The monomial corresponding to $M$ is the product

$$
\mathbf{x}^{M}=\prod_{m \in M} x_{m}
$$

having degree equal to the cardinality of $M$. For example, if $M=\{\{1,1,2$, $4,4,4\}$ then

$$
\mathbf{x}^{M}=x_{1}^{2} x_{2} x_{4}^{3} .
$$

The $k$ th elementary symmetric function is

$$
e_{k}\left(x_{1}, x_{2}, \ldots, x_{n}\right)=\sum_{S} x^{s}
$$

where the sum is over all subsets of $\{1,2, \ldots, n\}$ of cardinality $k$. Alternatively, it is the sum of all square-free monomials of degree $k$ in the variables $x_{1}, x_{2}, \ldots, x_{n}$. We will usually abbreviate $e_{k}\left(x_{1}, x_{2}, \ldots, x_{n}\right)$ to $e_{k}(n)$. To illustrate,

$$
e_{2}(3)=x_{1} x_{2}+x_{1} x_{3}+x_{2} x_{3} .
$$

The $k$ th complete homogeneous symmetric function is

$$
h_{k}\left(x_{1}, x_{2}, \ldots, x_{n}\right)=h_{k}(n)=\sum_{M} x^{M}
$$

where the sum is over all multisets of cardinality $k$ with elements from $\{1,2$, $\ldots, n\}$. It is the sum of all possible monomials of degree $k$ in $x_{1}, x_{2}, \ldots, x_{n}$. As an example,

$$
h_{2}(3)=x_{1} x_{2}+x_{1} x_{3}+x_{2} x_{3}+x_{1}^{2}+x_{2}^{2}+x_{3}^{2} .
$$

It is easy to see from the definitions that the elementary and complete homogeneous symmetric functions satisfy the following recurrence relations and boundary conditions.

Proposition 2.1. For $k \in \mathbf{Z}$ we have $e_{k}(0)=h_{k}(0)=\delta_{0, k}$. Further, for all $n \geq 1$,

1. $e_{k}(n)=e_{k}(n-1)+x_{n} e_{k-1}(n-1)$,

2. $h_{k}(n)=h_{k}(n-1)+x_{n} h_{k-1}(n)$.

Comparing this proposition with the definitions of the $q$-binomial coefficients and $q$-Stirling numbers a simple induction proves

Corollary 2.2. For $n, k \in \mathbf{N}$,

1. $\left[\begin{array}{l}n \\ k\end{array}\right]=q^{-\left(\begin{array}{c}k \\ 2\end{array}\right)} e_{k}\left(1, q, q^{2}, \ldots, q^{n-1}\right)=h_{k}\left(1, q, q^{2}, \ldots, q^{n-k}\right)$,

2. $c[n, k]=e_{n-k}([1],[2], \ldots,[n-1])$,

3. $S[n, k]=h_{n-k}([1],[2], \ldots,[k])$.

We now state a very special case of the Jacobi-Trudi Theorem. 
Proposition 2.3. If $0<k \leq l$ then the following determinants are polynomials in $\mathbf{N}[\mathbf{x}]$

1 .

2.

$$
\left|\begin{array}{cc}
e_{l}(n) & e_{l+1}(n) \\
e_{k-1}(n) & e_{k}(n)
\end{array}\right|
$$

$$
\left|\begin{array}{cc}
h_{l}(n) & h_{l+1}(n) \\
h_{k-1}(n) & h_{k}(n)
\end{array}\right| \text {. }
$$

It follows immediately from Corollary 2.2 and Proposition 2.3 that

Corollary 2.4. The following sequences are strongly q-log concave

$$
\left(q^{\left(\begin{array}{l}
k \\
2
\end{array}\right)}\left[\begin{array}{l}
n \\
k
\end{array}\right]\right)_{k \geq 0}, \quad\left(\left[\begin{array}{l}
n \\
k
\end{array}\right]\right)_{n \geq 0}, \quad(c[n, k])_{k \geq 0}, \quad(S[n, k])_{n \geq 0} .
$$

Thus three of the sequences from Theorem 1.1 can be handled using JacobiTrudi determinants. The rest will be taken care of by our analogs.

Note that in Proposition 2.3, the number of variables for each determinant entry is fixed while the degree varies. This raises the question: what happens if the degree is constant while the number of variables changes, or if both vary? These determinants no longer have nonnegative coefficients (in fact, they are no longer symmetric functions) but there is a sense in which they can be considered positive. The important concept in this connection is the definition of a standard partial order on the polynomial ring $\mathbf{R}[\mathbf{x}]$.

Definition 2.5. A partial order $\leq$ on $\mathbf{R}[\mathbf{x}]$ is standard if

1. $f(\mathbf{x}) \in \mathbf{R}^{+}[\mathbf{x}] \Rightarrow f(\mathbf{x}) \geq 0$,

2. $f(\mathbf{x}) \leq g(\mathbf{x}) \Rightarrow f(\mathbf{x})+h(\mathbf{x}) \leq g(\mathbf{x})+h(\mathbf{x})$ for all $h(\mathbf{x}) \in \mathbf{R}[\mathbf{x}]$,

3. $f(\mathbf{x}) \leq g(\mathbf{x}) \Rightarrow f(\mathbf{x}) h(\mathbf{x}) \leq g(\mathbf{x}) h(\mathbf{x})$ for all $h(\mathbf{x}) \geq 0$.

For example, the relation $\leq_{q}$ defined in the last section is a standard partial order on $\mathbf{R}[q]$. More generally, $\leq_{\mathbf{x}}$ defined by

$$
f(\mathbf{x}) \leq_{\mathbf{x}} g(\mathbf{x}) \text { if and only if } g(\mathbf{x})-f(\mathbf{x}) \in \mathbf{R}^{+}[\mathbf{x}]
$$

is standard.

Also, given any partial order $\leq$ on $R[\mathbf{x}]$ we can define strong log concavity with respect to $\leq$ as in equation (1) with $\leq_{q}$ replaced by $\leq$ and $q$ replaced by $\mathbf{x}$. With this convention, Proposition 2.3 can be reformulated.

$\left(e_{k}(n)\right)_{k \geq 0}$ and $\left(h_{k}(n)\right)_{k \geq 0}$ are strongly log concave with respect to $\leq_{\mathbf{x}}$. We can now state our principal result.

Theorem 2.6. If $\leq$ is standard and the sequence $\left(x_{n}\right)_{n \geq 0}$ is strongly log concave with respect to $\leq$, then so are the sequences

1. for fixed $k:\left(e_{k}(n)\right)_{n \geq 0}$ and $\left(h_{k}(n)\right)_{n \geq 0}$,

2. for fixed $n, k:\left(e_{k-j}(n+j)\right)_{j \geq 0}$ and $\left(h_{k-j}(n+j)\right)_{j \geq 0}$.

To prove this theorem, we will use the machinery of Gessel and Viennot [G-V $85]$.

\section{Gessel-VienNot Paths}

Consider a lattice path $P$ in $\mathbf{Z} \times \mathbf{Z}$ which uses only steps, $s$ of unit length north $(\mathrm{N})$ or east $(\mathrm{E})$. For example, we show such a path in Figure 1. 


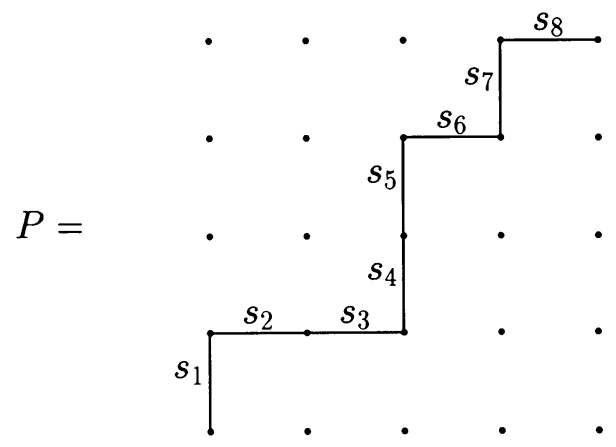

Figure 1. A lattice path

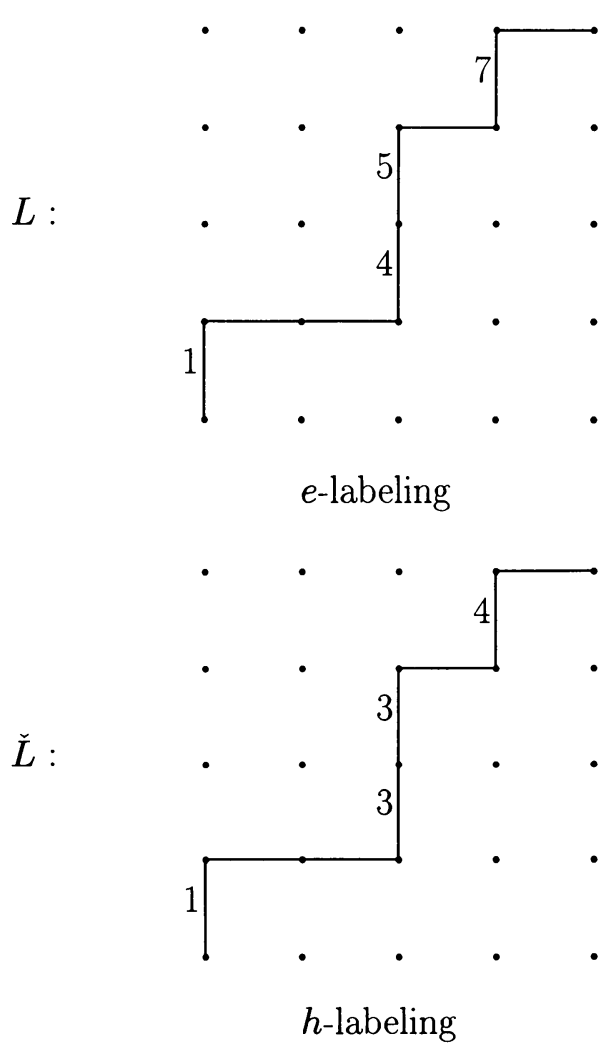

FIGURE 2. Labelings of a path

Let $P=s_{1}, s_{2}, \ldots, s_{n}$. We will label the northern steps of $P$ using one of two labelings. If $s_{i}$ is a step north then the $e$-labeling assigns the label

$$
L\left(s_{i}\right)=i
$$

while the $h$-labeling gives the label

$$
\check{L}\left(s_{i}\right)=\left(\text { the number of eastern } s_{j} \text { preceding } s_{i}\right)+1 \text {. }
$$

Intuitively, in the $h$-labeling all the northern steps on the line through the origin 
of $P$ are labeled 1, all those on the line one unit to the right are labeled 2, etc. Figure 2 displays the $e$-and $h$-labelings for the path in Figure 1 . Note that these labelings are invariant under translation.

Associated with each path, $P$, are two monomials corresponding to the two labelings, namely

$$
\mathbf{x}^{P}=\prod_{s_{i} \in P} x_{L\left(s_{i}\right)} \quad \text { and } \quad \check{\mathbf{x}}^{P}=\prod_{s_{i} \in P} x_{\check{L}\left(s_{i}\right)}
$$

where each product is taken over those $s_{i}=N$. If $P$ has initial vertex $u$ and final vertex $v$ then we will write $u \stackrel{P}{\longrightarrow} v$. From these definitions we clearly have

$$
e_{k}(n)=\sum_{P} \mathbf{x}^{P}
$$

where the sum is over all paths $(a, b) \stackrel{P}{\longrightarrow}(a+n-k, b+k)$ for any fixed initial vertex $(a, b)$. Similarly

$$
h_{k}(n)=\sum_{P} \check{\mathbf{x}}^{P}
$$

where the sum is now over all paths of the form $(a, b) \stackrel{P}{\longrightarrow}(a+n-1, b+k)$.

The fundamental tool in the Gessel-Viennot theory is an involution on pairs of paths that we will call $l$.

Definition 3.1. Given $u_{1} \stackrel{P_{1}}{\longrightarrow} v_{1}$ and $u_{2} \stackrel{P_{2}}{\longrightarrow} v_{2}$ then define $l\left(P_{1}, P_{2}\right)=$ $\left(P_{1}^{\prime}, P_{2}^{\prime}\right)$ where

1. if $P_{1} \cap P_{2}=\varnothing$ then $P_{1}^{\prime}=P_{1}$ and $P_{2}^{\prime}=P_{2}$,

2. if $P_{1} \cap P_{2} \neq \varnothing$ then

$$
P_{1}^{\prime}=u_{1} \stackrel{P_{1}}{\longrightarrow} v_{0} \stackrel{P_{2}}{\longrightarrow} v_{2} \text { and } P_{2}^{\prime}=u_{2} \stackrel{P_{2}}{\longrightarrow} v_{0} \stackrel{P_{1}}{\longrightarrow} v_{1}
$$

where $v_{0}$ is the last (NE-most) vertex of $P_{1} \cap P_{2}$, i.e., switch the portions of $P_{1}$ and $P_{2}$ after $v_{0}$.

By way of illustration, Figure 3 shows the effect of $l$ on a particular pair $P_{1}$ (solid lines) and $P_{2}$ (dashed lines).

Now fix an ordered pair of initial vertices $u_{1}, u_{2}$ and an ordered pair of final ones $v_{1}, v_{2}$. Assuming that path $P_{i}$ always departs from $u_{i}$ for $i=1,2$, define the sign of the pair $P_{1}, P_{2}$ to be

$$
(-1)^{P_{1} P_{2}}= \begin{cases}+1 & \text { if } u_{1} \stackrel{P_{1}}{\longrightarrow} v_{1} \text { and } u_{2} \stackrel{P_{2}}{\longrightarrow} v_{2}, \\ -1 & \text { if } u_{1} \stackrel{P_{1}}{\longrightarrow} v_{2} \text { and } u_{2} \stackrel{P_{2}}{\longrightarrow} v_{1}\end{cases}
$$

Also let the e-monomial of the pair be

$$
\mathbf{x}^{P_{1} P_{2}}=\mathbf{x}^{P_{1}} \mathbf{x}^{P_{2}}
$$

and similarly for the $h$-monomial. It follows that

$$
\left|\begin{array}{cc}
e_{k}(n) & e_{k}(n+1) \\
e_{k}(n-1) & e_{k}(n)
\end{array}\right|=\sum_{P_{1}, P_{2}}(-1)^{P_{1} P_{2}} \mathbf{x}^{P_{1} P_{2}}
$$



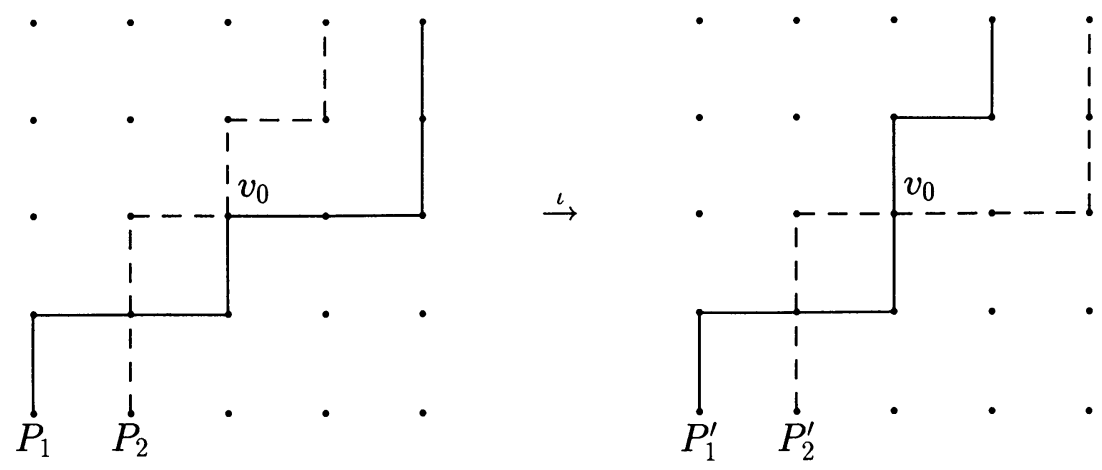

FIGURE 3. The involution $l$

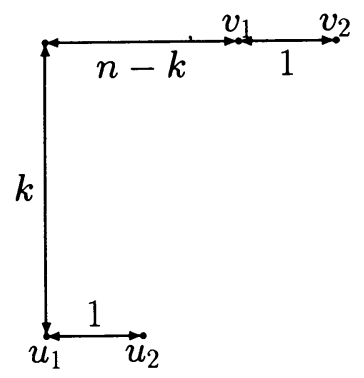

FIGURE 4. Initial and final vertices

where the sum is over all pairs $P_{1}, P_{2}$ having

$$
u_{1}=(0,0) ; \quad u_{2}=(1,0) ; \quad v_{1}=(n-k, k) ; \quad v_{2}=(n-k+1, k) \text {. }
$$

This configuration is given schematically in Figure 4.

\section{MAIN RESUltS}

We are now in a position to demonstrate Theorem 2.6. For ease of reference, we restate the portion we will prove at this juncture.

Theorem 4.1. Fix $k \in \mathbf{N}$. If $\leq$ is standard and the sequence $\left(x_{n}\right)_{n \geq 0}$ is strongly log concave with respect to $\leq$, then so are the sequences

$$
\left(e_{k}(n)\right)_{n \geq 0} \text { and }\left(h_{k}(n)\right)_{n \geq 0} .
$$

Proof. We will show that

$$
e_{k}(n)^{2}-e_{k}(n-1) e_{k}(n+1) \geq 0
$$

and then indicate what modifications need to be made for the general case and for the $h_{k}(n)$.

Let $u_{1}, u_{2}, v_{1}$ and $v_{2}$ be situated as indicated at the end of the previous section. Thus it suffices to prove that if $P_{1}, P_{2}$ is a path pair with sign -1 , then $l\left(P_{1}, P_{2}\right)=\left(P_{1}^{\prime}, P_{2}^{\prime}\right)$ (which must have sign +1$)$ satisfies

$$
\mathbf{x}^{P_{1}^{\prime} P_{2}^{\prime}}-\mathbf{x}^{P_{1} P_{2}} \geq 0 \text {. }
$$


Since $\left(x_{n}\right)_{n \geq 0}$ is strongly log concave, the above inequality must hold because we can write the two monomials involved as

$$
\begin{aligned}
\mathbf{x}^{P_{1} P_{2}} & =\mathbf{y} \prod_{i} x_{a_{i}-1} x_{b_{i}+1}, \\
\mathbf{x}^{P_{1}^{\prime} P_{2}^{\prime}} & =\mathbf{y} \prod_{i} x_{a_{i}} x_{b_{i}},
\end{aligned}
$$

where $\mathbf{y}$ is a fixed monomial and $a_{i} \leq b_{i}$ for all $i$. In particular, $\mathbf{y}$ corresponds to the portions of $P_{1}$ and $P_{2}$ which are fixed by $l$ (everything SW of $v_{0}$ ) while the product represents the parts that are switched. The indices change as they do due to the fact that $P_{1}$ starts one unit west of $P_{2}$.

To show that

$$
e_{k}(m) e_{k}(n)-e_{k}(m-1) e_{k}(n+1) \geq 0
$$

for $m \leq n$, merely use the initial point $u_{2}=(n-m+1,0)$. The corresponding inequality for the complete homogeneous symmetric functions follows from choosing $v_{1}=(n-1, k)$ and $v_{2}=(n, k)$ (with $u_{2}$ as in the previous sentence).

For example, if the path pair and its image are as given in Figure 5, then

$$
\begin{aligned}
& \mathbf{x}^{P_{1} P_{2}}=x_{1}^{2} x_{2} x_{4}\left(x_{4} x_{7}\right)\left(x_{6} x_{8}\right), \\
& \mathbf{x}_{1}^{P_{1}^{\prime} P_{2}^{\prime}}=x_{1}^{2} x_{2} x_{4}\left(x_{5} x_{6}\right)\left(x_{7} x_{7}\right),
\end{aligned}
$$

where the pairs of the products in (4) and (3) are enclosed in parentheses.

As an immediate corollary we obtain several more parts of Theorem 1.1.

Corollary 4.2. The following sequences are all strongly q-log concave,

$$
\left(\left[\begin{array}{l}
n \\
k
\end{array}\right]\right)_{n \geq 0}, \quad(c[n+j, k+j])_{j \geq 0}, \quad(S[n+j, k+j])_{j \geq 0} .
$$

To examine the case where both $k$ and $n$ vary, we will borrow an idea from Butler [But 90] which has also been used by Leroux [Ler 90]. Let $\tau_{d}$ denote translation by one unit in the direction $d=\mathrm{N}$ or $\mathrm{E}$. Apply this function to a path pair by acting on the first component only:

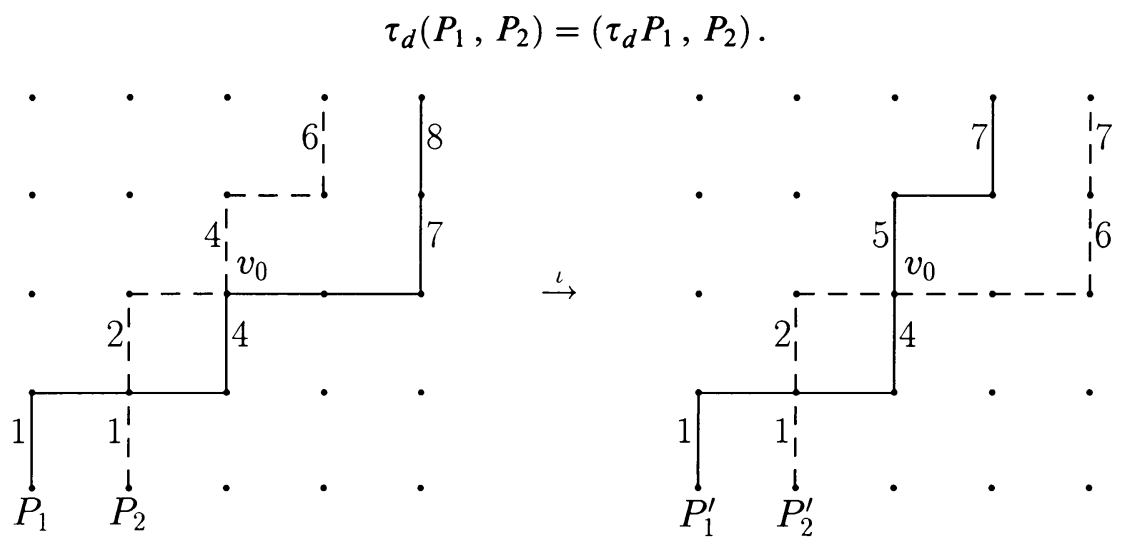

FIGURE 5. The involution of Theorem 4.1 


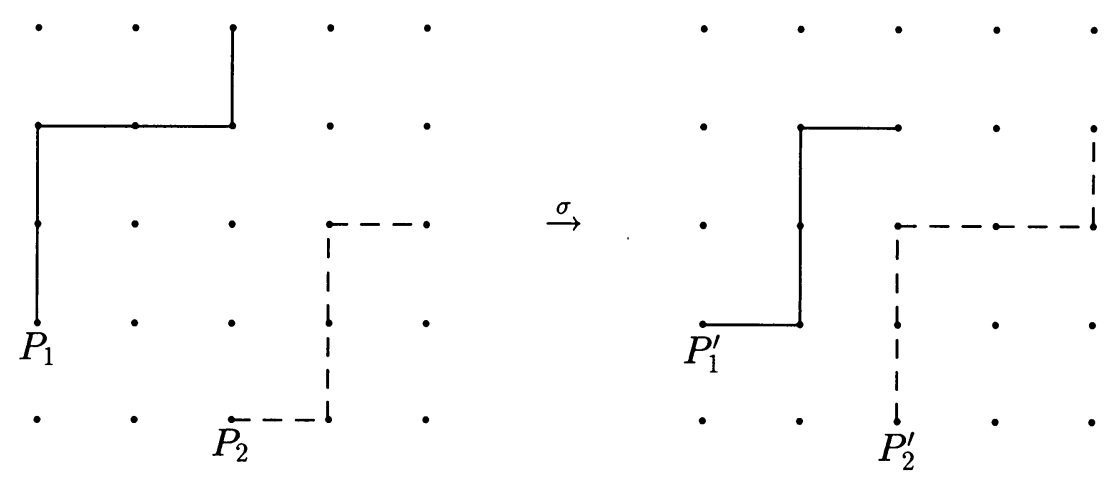

Figure 6. The INVOLUTION $\sigma$

Also consider the switching involution $\sigma$ defined by

$$
\sigma\left(P_{1}, P_{2}\right)=\left(P_{1}^{\prime}, P_{2}^{\prime}\right)
$$

where $P_{1}^{\prime}$ is the path $P_{2}$ translated to start at the initial point of $P_{1}$ and vice versa for $P_{2}^{\prime}$. An example is given in Figure 6. Maps of the form $\tau_{d}^{-1} l \tau_{d}$ and $\sigma \tau_{d}^{-1} l \tau_{d}$ (functions compose right to left) are the injections that we will need.

Theorem 4.3. Fix $k, n \in \mathbf{N}$. If $\leq$ is standard and the sequence $\left(x_{n}\right)_{n \geq 0}$ is strongly log concave with respect to $\leq$, then so are the sequences

$$
\left(e_{k-j}(n+j)\right)_{j \geq 0} \text { and }\left(h_{k-j}(n+j)\right)_{j \geq 0} .
$$

Proof. This time we will demonstrate

$$
h_{k}(n)^{2}-h_{k-1}(n+1) h_{k+1}(n-1) \geq 0,
$$

giving the necessary modifications for the other cases at the end.

Use initial points

$$
u_{1}=(0,0) ; \quad u_{2}=(1,-1)
$$

and final ones

$$
v_{1}=(n-1, k) ; \quad v_{2}=(n, k-1)
$$

as represented in Figure 7.

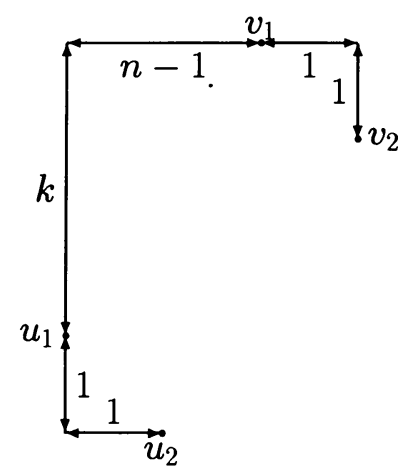

FIGURE 7. More initial and final vertices 
By our selection of the $u_{i}$ and $v_{i}$, the left side of equation (5) is the generating function for corresponding path pairs with the $h$-labeling.

Consider the composition

$$
\left(P_{1}, P_{2}\right) \stackrel{\tau_{N}^{-1} l \tau_{N}}{\longrightarrow}\left(P_{1}^{\prime}, P_{2}^{\prime}\right) \stackrel{\sigma \tau_{E}^{-1} l \tau_{E}}{\longrightarrow}\left(P_{1}^{\prime \prime}, P_{2}^{\prime \prime}\right) .
$$

Assume for the rest of the proof that $(-1)^{P_{1} P_{2}}=-1$, i.e., $u_{1} \stackrel{P_{1}}{\longrightarrow} v_{2}$ and $u_{2} \stackrel{P_{2}}{\longrightarrow} v_{1}$. Then $\left(P_{1}^{\prime \prime}, P_{2}^{\prime \prime}\right)$ has the same set of initial and final vertices with sign +1 . (The presence of the switching operator is to ensure that this continues to hold in the more general case.)

Since our maps are all injections, we will be done if

$$
\check{\mathbf{x}}_{1}^{\prime \prime} P_{2}^{\prime \prime}-\check{\mathbf{x}}^{P_{1} P_{2}} \geq 0 \text {. }
$$

But

$$
\check{\mathbf{x}}^{P_{1} P_{2}} \leq \check{\mathbf{x}}^{\prime} P_{2}^{\prime}
$$

since the sequence $\left(x_{n}\right)_{n \geq 0}$ is strongly log concave and path $P_{1}$ starts on a vertical line one unit west of $P_{2}$ (which is not affected by north/south translations). Note that the northward translation of $P_{1}$ is necessary so that the resulting pair of paths both end on the same horizontal line. This insures that, in the notation of the previous theorem, each $x_{a_{i}}$ has a matching $x_{b_{i}}$. Finally

$$
\check{\mathbf{x}}_{1}^{\prime} P_{2}^{\prime}=\breve{\mathbf{x}}^{P_{1}^{\prime \prime} P_{2}^{\prime \prime}}
$$

for any values of the $x_{n}$ because $\tau_{E}\left(P_{1}^{\prime}\right)$ starts on the same vertical line as $P_{2}^{\prime}$. For the general case

$$
h_{k}(n) h_{l}(m)-h_{k-1}(n+1) h_{l+1}(m-1) \geq 0
$$

where $k \leq l$ and $k+n=l+m$, the second initial point must be changed to $u_{2}=(n-m+1, m-n-1)$. It is also necessary to translate $P_{1}^{\prime}$ by $\tau_{E}^{n-m+1} \tau_{N}^{m-n}$ before performing $l$ (and then translating back by the same amount). Thus the translated path will start one unit due north of $P_{2}^{\prime}$, guaranteeing that $\check{\mathbf{x}}_{1}^{P_{1}^{\prime \prime} P_{2}^{\prime \prime}}$ will be a term of $h_{k}(n) h_{l}(m)$.

To show that (9) holds with the complete homogeneous symmetric functions replaced by elementaries, use

$$
\begin{array}{ll}
u_{1}=(0,0) ; & u_{2}=(2(n-m+1), m-n-1) ; \\
v_{1}=(n-k, k) ; & v_{2}=(n-k+2, k-1) .
\end{array}
$$

The translation used for $P_{1}$ should now be $\tau_{E}^{-1} \tau_{N}$ so that the translate takes exactly one more step than $P_{2}$ to get to one of their intersections. Lastly, $P_{1}^{\prime}$ should be translated by $\tau_{E}^{2 n-2 m+1} \tau_{N}^{m-n}$ prior to applying $l$.

Note that if $\left(P_{1}, P_{2}\right)$ has sign -1 in $(6)$, then $\check{\mathbf{x}}^{P_{1} P_{2}}, \check{\mathbf{x}}_{1}^{\prime} P_{2}^{\prime}$ and $\check{\mathbf{x}}^{\prime \prime} P_{2}^{\prime \prime}$ are terms in $h_{k-1}(n+1) h_{k+1}(n-1), h_{k-1}(n) h_{k+1}(n)$ and $h_{k}(n)^{2}$ respectively. It follows from the proof of (7) and (8) that

$$
h_{k-1}(n+1) h_{k+1}(n-1) \leq h_{k-1}(n) h_{k+1}(n)
$$

and

$$
h_{k-1}(n) h_{k+1}(n) \leq h_{k}(n)^{2} \text {. }
$$


(Of course, together these imply (5).) Intuitively, the map $\left(P_{1}, P_{2}\right) \rightarrow\left(P_{1}^{\prime}, P_{2}^{\prime}\right)$ corrects the number of variables while $\left(P_{1}^{\prime}, P_{2}^{\prime}\right) \rightarrow\left(P_{1}^{\prime \prime}, P_{2}^{\prime \prime}\right)$ corrects the degree. More generally we have the following result whose proof, being similar to that of the previous theorem, is omitted. Dennis Stanton has pointed out [private communication] that parts 1 and 2 of this theorem are merely special cases of the Jacobi-Trudi determinant for flagged Schur functions [Ges um, Wac 85]

Theorem 4.4. Fix natural numbers $k \leq l$ and $m \leq n$. If $\leq i$ standard then

1. $e_{k-1}(n) e_{l+1}(m) \leq e_{k}(n) e_{l}(m)$,

2. $h_{k-1}(n) h_{l+1}(m) \leq h_{k}(n) h_{l}(m)$.

If, the addition, the sequence $\left(x_{n}\right)_{n \geq 0}$ is strongly log concave with respect to $\leq$, then

3. $e_{k}(n+1) e_{l}(m-1) \leq e_{k}(n) e_{l}(m)$,

4. $h_{k}(n+1) h_{l}(m-1) \leq h_{k}(n) h_{l}(m)$.

We can also complete the list of sequences from Theorem 1.1 shown to be $q$-log concave.

Corollary 4.5. The following sequences are all strongly q-log concave $\left(q^{\left(\begin{array}{c}k \\ 2\end{array}\right)}\left[\begin{array}{c}n-k \\ k\end{array}\right]\right)_{k \geq 0}, \quad\left(\left[\begin{array}{l}n \\ k\end{array}\right]\right)_{k \geq 0}, \quad(c[n+j, k+2 j])_{j \geq 0}, \quad(S[n, k])_{k \geq 0}$.

\section{PF SEQUENCES}

Although the definition of a PF sequence is usually stated for sequences of real numbers [Bre 88], there is nothing to prevent us from generalizing it to polynomials. Let $\leq$ be a partial order on $\mathbf{R}[\mathbf{x}]$. A sequence $\left(f_{n}\right)_{n \geq 0}$ where each $f_{n} \in \mathbf{R}[\mathbf{x}]$ is $P F$ with respect to $\leq$ if all minors of the infinite matrix

$$
\left(f_{j-i}\right)_{i, j \geq 0}=\left(\begin{array}{cccc}
f_{0} & f_{1} & f_{2} & \cdots \\
0 & f_{0} & f_{1} & \cdots \\
0 & 0 & f_{0} & \cdots \\
\vdots & \vdots & \vdots & \ddots
\end{array}\right)
$$

are $\geq 0$. It is a consequence of the full Jacobi-Trudi Theorem that

$$
\left(e_{k}(n)\right)_{k \geq 0} \text { and }\left(h_{k}(n)\right)_{k \geq 0} \text { are PF with respect to } \leq_{\mathbf{x}} \text {. }
$$

Note that if a sequence is $\mathrm{PF}$ with respect to $\leq$ then it is strongly log concave since the $2 \times 2$ minors include the determinants for log concavity.

The Gessel-Viennot machine takes care of $d \times d$ determinants by using $d$ tuples of paths. Let $u_{1}, u_{2}, \ldots, u_{d}$ and $v_{1}, v_{2}, \ldots, v_{d}$ be fixed sets of initial and final vertices. Consider a family of paths $\mathscr{P}=\left(P_{1}, P_{2}, \ldots, P_{d}\right)$ where, for each $i$,

$$
u_{i} \stackrel{P_{i}}{\longrightarrow} v_{\sigma i}
$$

for some $\sigma \in S_{d}$, the symmetric group on $d$ elements. Define

(the sign of $\sigma$ ) and

$$
(-1)^{\mathscr{P}}=(-1)^{\sigma}
$$

with a similar definition for $\check{\mathbf{x}}^{\mathscr{P}}$.

$$
\mathbf{x}^{\mathscr{P}}=\prod_{i} \mathbf{x}^{P_{i}}
$$

The involution $l$ can now be extended to this situation. 


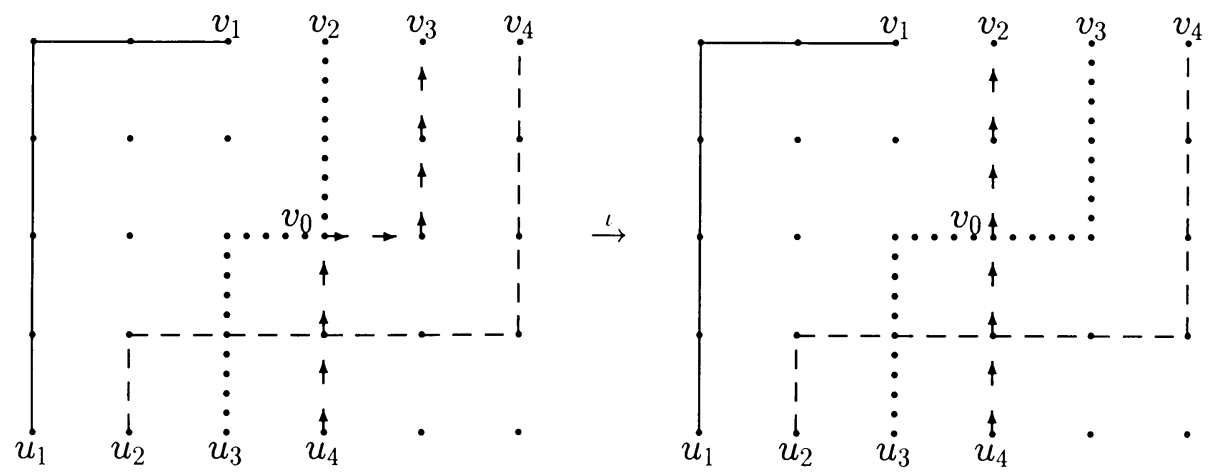

Figure 8. The involution $l$ for $d$-tuples

Definition 5.1. Given $\mathscr{P}$ as above, define $\imath^{\mathscr{P}}=\mathscr{P}^{\prime}$ where

1. if $P_{i} \cap P_{j}=\varnothing$ for all $i, j$ then $\mathscr{P}=\mathscr{P}^{\prime}$,

2. else find the smallest index $j$ such that the path $P_{l}=P_{\sigma^{-1} j}$ to $v_{j}$ intersects some other path. Let $v_{0}$ be the last (NE-most) intersection on $P_{l}$ and let $P_{m}$ be the other path through $v_{0}$. (If there is more than one, choose $P_{m}$ so that $\sigma m$ is minimal.) Now define

$$
\mathscr{P}^{\prime}=\mathscr{P} \text { with }\left(P_{l}, P_{m}\right) \text { replaced by } l\left(P_{l}, P_{m}\right) \text {. }
$$

It is not hard to verify that this is really an involution (although it is crucial in the proof that we define $l$ in terms of the final vertices $v_{j}$ rather than the initial $u_{i}$ ). An example is given in Figure 8.

The analog of Theorem 4.1 in this setting is

Theorem 5.2. Fix $k \in \mathbf{N}$. If $\leq$ is standard and $x_{k-1} x_{l+1}=x_{k} x_{l}$ for $0<k \leq l$, then the sequences $\left(e_{k}(n)\right)_{n \geq 0}$ and $\left(h_{k}(n)\right)_{n \geq 0}$ are PF with respect to $\leq$.

Proof. In the matrix $\left(e_{k}(j-i)\right)$, the minor with rows $i_{1}, i_{2}, \ldots, i_{d}$ and columns $j_{1}, j_{2}, \ldots, j_{d}$ counts paths with

$$
u_{c}=\left(i_{c}-i_{1}, 0\right) \text { and } v_{c}=\left(j_{c}-i_{1}-k, k\right)
$$

for all $1 \leq c \leq d$. Figure 9 illustrates this arrangement.

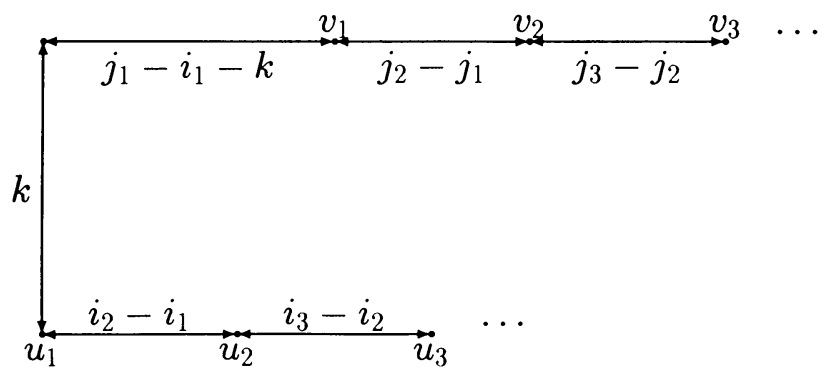

FIGURE 9. Initial and final vertices for Theorem 5.2 
Now given a family of paths $\mathscr{P}$ with sign -1 , we can apply $\imath$ to get a family $\mathscr{P}^{\prime}$ with sign +1 . The monomials for $\mathscr{P}$ and $\mathscr{P}^{\prime}$ are given by $\mathbf{y} \prod_{i} x_{a_{i}} x_{b_{i}}$ and $\mathbf{y} \prod_{i} x_{a_{i}-1} x_{b_{i}+1}$ or vice versa ( $\mathbf{y}$ fixed and $a_{i} \leq b_{i}$ for all $i$ ). It is for this reason that we need the strong assumption about the sequence $\left(x_{n}\right)_{n \geq 0}$.

For the $h_{k}(n)$, the final vertices are changed to

$$
v_{c}=\left(j_{c}-i_{1}-1, k\right)
$$

for all $c$. Everything else remains the same.

It is a pity that we are forced to strengthen the hypothesis in passing from Theorem 4.1 to Theorem 5.2. Because of this, we can only get a corollary for the $q$-binomial coefficients.

Corollary 5.3. Fix $k \in \mathbf{N}$. Then the sequence

$$
\left(\left[\begin{array}{l}
n \\
k
\end{array}\right]\right)_{n \geq 0}
$$

is $P F$ with respect to $\leq_{q}$.

Unfortunately, the methods used to prove Theorem 4.3 cannot be extended to the PF case. We will discuss this more fully in the next section.

On the bright side, there are several other infinite matrices that also have every minor nonnegative even though they are not associated with any sequence. In what follow, the variables $i$ and $j$ always give the row and column of the matrix. Note that this result generalizes Theorem 4.4.

Theorem 5.4. Fix $k, l, m \in \mathbf{N}$. Then all minors of the following matrices are in $\mathbf{N}[\mathbf{x}]$, i.e., are $\geq 0$ for any standard partial order.

1. $\left(e_{j-k i}(l i+m)\right)$,

2. $\left(h_{j-k i}(l i+m)\right)$.

If the sequence $\left(x_{n}\right)_{n \geq 0}$ is strongly log concave with respect to $\leq$, then all $2 \times 2$ minors of the following matrices are nonnegative. If in addition, $x_{k-1} x_{l+1}=x_{k} x_{l}$ for all $0<k \leq l$, then all minors of these matrices are nonnegative.

3. $\left(e_{l j+m}(i-k j)\right)$,

4. $\left(h_{l j+m}(i-k j)\right)$.

Proof. All parts of the theorem follow by applying $l$ to a given family of paths, so we need only give the initial and final vertices for each case. In these definitions, $c$ varies between 1 and $d$ (where $d$ is the number of paths) while $i_{c}$ and $j_{c}$ stand for the $c$ th row and column of the minor.

1. $u_{c}=\left(j_{c}-j_{1}, j_{1}-j_{c}\right) ; v_{c}=\left((k+l) i_{c}-j_{1}+m, j_{1}-k i_{c}\right)$;

2. $u_{c}=\left(0, j_{1}-j_{c}\right) ; v_{c}=\left(l i_{c}+m-1, j_{1}-k i_{c}\right)$;

3. $u_{c}=\left((k+l)\left(j_{c}-j_{1}\right), l\left(j_{1}-j_{c}\right)\right) ; v_{c}=\left(i_{c}-(k+l) j_{1}-m, l j_{1}+m\right)$;

4. $u_{c}=\left(k\left(j_{c}-j_{1}\right), l\left(j_{1}-j_{c}\right)\right) ; v_{c}=\left(i_{c}-k j_{1}-1, l j_{1}+m\right)$.

Specializing to our $q$-analogs, we obtain

Corollary 5.5. Fix $k, l, m \in \mathbf{N}$. All the minors of the following matrices are in $\mathbf{N}[q]$.

1. $\left(q^{\left(\begin{array}{c}j-k i \\ 2\end{array}\right)}\left[\begin{array}{c}l i+m \\ j-k i\end{array}\right]\right) ;\left(q^{\left(\begin{array}{c}(j+m \\ 2\end{array}\right)}\left[\begin{array}{c}i-k j \\ l j+m\end{array}\right]\right) ;$

2. $\left(\left[\begin{array}{c}j+(l-k) i+m \\ j-k i\end{array}\right]\right) ;\left(\left[\begin{array}{c}i+(l-k) j+m \\ l j+m\end{array}\right]\right)$;

3. $(c[l i+m,(k+l) i-j+m]) ;(S[(l-k) i+j+m, l i+m])$;

In addition, all $2 \times 2$ minors of the following matrices are nonnegative.

4. $(c[i-k j, i-(k+l) j+m]) ;(S[i+(l-k) j+m, i-k j])$. 


\section{COMMENTS AND OPEN QUESTIONS}

This work raises several interesting problems listed below.

1. Proposition 2.3 and Theorems 4.1 and 4.3 show log concavity of symmetric function sequences when $n, k$ or $n+k$ is constant. It would be interesting to see for which linear relations between $n$ and $k$ we obtain log concave sequences. Perhaps there is some analog of Theorem 5.4 in this regard.

2. We have already remarked that for polynomials, log concavity and strong $\log$ concavity are not equivalent (unlike the situation for sequences of positive real numbers). Is there a nice condition that could be added to log concavity in the general case which would then imply strong log concavity?

3 . The reader may have noticed that there is a sequence missing from Theorem 1.1, namely $(c[n, k])_{n>0}$. It is absent because it is not $q$-log concave even in the case $q=1$ (as can be seen by taking $k=1$ ). Pierre Leroux [Ler 90] conjectured that an "almost" log-concave condition holds, namely

$$
c[n-1, k] c[n+1, k] \leq[2] c[n, k]^{2} .
$$

this was proved when $q=1$ by Anne de Médicis [Méd 89]. On the basis of (11), we were led to conjecture the following result which has been recently proved by Laurent Habsieger [Hab pr].

Theorem 6.1. Let $\leq$ be standard. Suppose there exists a fixed $b \in \mathbf{R}[\mathbf{x}]$ such that $b x_{n} \geq x_{n+1}$ for all $n \geq 1$. Then for all $n \geq 0$ and $0<k \leq l$ we have

$$
e_{k-1}(n+k-1) e_{l+1}(n+l+1) \leq b^{l-k+1} e_{k}(n+k) e_{l}(n+l) .
$$

Note that Leroux's equation is the special case where $k=l, x_{n}=[n]$ and $b=[2]$. It is possible to find an analogous theorem for "almost" PF sequences?

4. There are a number of reasons why the proof of Theorem 4.3 does not generalize to the PF case. For one thing, the compositions are not injective when applied to $d$-tuples. This is because the translations and switching operators create and destroy the intersections which tag the pair of paths to be used. Another problem is that the path pair through $v_{0}$ may have sign +1 even though $\mathscr{P}$ has sign -1 . In this case applying these maps will not preserve the terminal points of the pair.

Nevertheless, we conjecture that the following is true.

Conjecture 6.2. Fix $k, n \in \mathbf{N}$. If $\leq$ is standard and $x_{k-1} x_{l+1}=x_{k} x_{l}$ for all $0<k \leq l$, then the sequence $\left(e_{k-j}(n+j)\right)_{j \geq 0}$ is PF with respect to $\leq$.

5. It follows from $(10)$ in $\S 5$ that the following sequences are $q$-PF, i.e., PF with respect to $\leq_{q}$ :

$$
\left(\left[\begin{array}{l}
n \\
k
\end{array}\right]\right)_{n \geq 0}, \quad\left(q^{\left(\begin{array}{c}
k \\
2
\end{array}\right)}\left[\begin{array}{l}
n \\
k
\end{array}\right]\right)_{k \geq 0}, \quad(c[n, k])_{k \geq 0}, \quad(S[n, k])_{n \geq 0} .
$$

The first of these was also derived from Theorem 5.2. It would be interesting to find out which of the other sequences in Theorem 1.1 are also $q$-PF. Christian Krattenthaler has pointed out that

$$
\left(\left[\begin{array}{l}
n \\
k
\end{array}\right]\right)_{k \geq 0}
$$


is not $q-\mathrm{PF}$ since

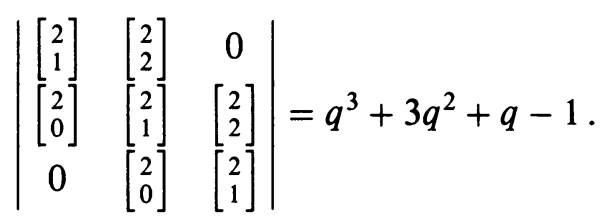

Thus Conjecture 6.2 cannot be true for the sequence $\left(h_{k-j}(n+j)\right)_{j \geq 0}$ since it becomes (12) under the usual substitution. However, the elementary symmetric function sequence in the conjecture specializes to

$$
\left(q^{\left({ }^{(-j}\right)}\left[\begin{array}{l}
n+j \\
k-j
\end{array}\right]\right)_{j \geq 0}
$$

and we have checked by computer that all $3 \times 3$ minors for this sequence are polynomials with nonnegative coefficients for $n+k \leq 7$. John Stembridge has also computer-verified that all $3 \times 3$ minors of $(S[n+j, k+j])_{j \in N}$ are nonnegative for $1 \leq k, n-k \leq 5$. It would be nice to prove such a fact as a corollary to results like Theorem 5.2 and Conjecture 6.2, however their hypotheses would have to be weakened to apply to the Stirling numbers.

6 . Let $\lambda=\left(\lambda_{1}, \lambda_{2}, \ldots, \lambda_{d}\right)$ be a partition of $n$, i.e., a sequence of weakly decreasing nonnegative integers whose sum is $n$. The Jacobi-Trudi determinants are the $d \times d$ determinants

$$
\left|e_{\lambda_{i}+j-i}\right| \text { and }\left|h_{\lambda_{i}+j-i}\right| .
$$

Let $S_{n}$ denote the symmetric group on $n$ letters. The Schur function corresponding to $\lambda, s_{\lambda}(n)$, is the cycle indicator generating function for the irreducible character of $S_{n}$ associated with $\lambda$. In fact

$$
s_{\lambda}(n)=\left|h_{\lambda_{i}+j-i}\right|
$$

and

$$
s_{\lambda^{\prime}}(n)=\left|e_{\lambda_{i}+j-i}\right|,
$$

where $\lambda^{\prime}$ is the conjugate of $\lambda$. Thus both Jacobi-Trudi determinants are polynomials with nonnegative coefficients.

For any sequence $\left(f_{n}\right)_{n \geq 0}$ where each $f_{n} \in \mathbf{R}[\mathbf{x}]$ we can define the generalized Jacobi-Trudi determinant corresponding to a partition $\lambda$ as $\left|f_{\lambda_{i}+j-i}\right|$. Note that these are just the minors of $\left(f_{j-i}\right)$ obtained by taking adjacent columns. Clearly, if $\left(f_{n}\right)_{n \geq 0}$ is PF with respect to $\geq$ then all of its Jacobi-Trudi determinants are nonnegative. Brenti [Bre 88, Theorem 7.4.1] (and later Stembridge [private communication]) provided the following proof that the converse also holds. In fact, Price has shown [Pri 65] that a sequence is PF if and only if all minors obtained from adjacent rows and columns are nonnegative.

Theorem 6.3. Let $\leq$ be standard. Then $\left(f_{n}\right)_{n \geq 0}$ is $P F$ with respect to $\leq$ if and only if $\left|f_{\nu_{i}+j-i}\right| \geq 0$ for all partitions $\nu$.

Proof. It is only necessary to prove the "if" direction.

Suppose $\lambda=\left(\lambda_{1}, \lambda_{2}, \ldots, \lambda_{d}\right)$ and $\mu=\left(\mu_{1}, \mu_{2}, \ldots, \mu_{d}\right)$ are partitions such that $\lambda_{i} \geq \mu_{i}$ for all $i$. Then there is an associated skew Schur function, $s_{\lambda / \mu}(n)$, which is the generating function for an induced representation of $S_{n}$. We can also express this as a determinant

$$
s_{\lambda / \mu}(n)=\left|h_{\lambda_{i}-\mu_{j}+j-i}\right| .
$$


Now the ordinary Schur functions form a basis for the space of symmetric functions, so we can write

$$
s_{\lambda / \mu}(n)=\sum_{\nu} c_{\mu \nu}^{\lambda} s_{\nu}(n)
$$

for certain constants $c_{\mu \nu}^{\lambda} \in \mathbf{R}$. Furthermore, since the representation corresponding to $s_{\lambda / \mu}(n)$ is not virtual, we have $c_{\mu \nu}^{\lambda} \geq 0$ for all $\lambda, \mu, \nu$. Finally, substituting equations (13) and (15) into (16) we have a polynomial identity involving the complete homogeneous symmetric functions. But the $h_{m}(n)$ are algebraically independent, so we may replace them by arbitrary elements of $\mathbf{R}[\mathbf{x}]$ without disturbing the equality.

Now choose any minor $M$ of $\left(f_{j-i}\right)$, say with rows $i_{1}, i_{2}, \ldots, i_{d}$ and columns $j_{1}, j_{2}, \ldots, j_{d}$. Define partitions $\lambda$ and $\mu$ by

$$
\lambda_{k}=j_{d}-i_{k}-d+k \text { and } \mu_{l}=j_{d}-j_{l}-d+l .
$$

Then

$$
M=\left|f_{\lambda_{k}-\mu_{l}+l-k}\right|
$$

and so substituting $f_{m}$ for $h_{m}(n)$ in equation (16) we have expressed $M$ as a nonnegative linear combination of generalized Jacobi-Trudi determinants. The theorem follows.

7. The Schur function $s_{\lambda}(n)$ can also be interpreted as the ordinary generating function for semistandard Young tableaux of shape $\lambda[\operatorname{Mad} 79$, p. 42; Sag 90; Sag 91, p. 151]. This can be seen by interpreting the labels on each path of a nonintersecting $d$-tuple counted by a Jacobi-Trudi determinant as a row (in the case of equation (13)) or column (for (14)) of a tableau. It would be interesting to find a similar interpretation for the minors in Theorem 5.2.

\section{REFERENCES}

[Bre 88] F. Brenti, Unimodal, log-concave and Polya frequency sequences in combinatorics, Mem. Amer. Math. Soc., no. 413, 1989.

[But 90] L. M. Butler, The q-log concavity of q-binomial coefficients, J. Combin. Theory Ser. A 54 (1990), 54-63.

[Ges um] I. Gessel, Determinants and plane partitions, unpublished manuscript.

[G-V 85] I. Gessel and G. Viennot, Binomial determinants, paths, and hook length formulae, Adv. in Math. 58 (1985), 300-321.

[Hab pr] L. Habsieger, Inégalités entre fonctions symétriques élémentaires: applications à des problèmes d'unimodalité, preprint.

[Kra 89] C. Krattenthaler, On the q-log-concavity of Gaussian binomial coefficients, Monatsh. Math. 107 (1989), 333-339.

[Ler 90] P. Leroux, Reduced matrices and q-log concavity properties of q-Stirling numbers, J. Combin. Theory Ser. A 54 (1990), 64-84.

[Mad 79] I. G. Macdonald, Symmetric functions and Hall polynomials, Oxford Univ. Press, Oxford, 1979.

[Méd 89] A. de Médicis, Unimodalité et concavité logarithmique pour des suites de nombres classiques et leurs q-analogues, Masters thesis, Université du Québec à Montréal, Montréal, 1989.

[Pri 65] H. S. Price, Monotone and oscillating matrices applied to finite difference approximation, Ph.D. thesis, Case Institute of Technology, Cleveland, 1965. 
[Sag 88] B. E. Sagan, Inductive and injective proofs of log concavity results, Discrete Math. 68 (1988), 281-292.

[Sag 90] __ The ubiquitous Young tableau, Invariant Theory and Tableaux (Dennis Stanton, ed.), IMA Vol. Math. Appl. 19 (1990), 262-298.

[Sag 91] _ , The symmetric group: representations, combinatorial algorithms, and symmetric functions, Wadsworth \& Brooks/Cole, Pacific Grove, 1991.

[Sag ta] __, Inductive proofs of q-log concavity, Discrete Math. (to appear).

[Sta 89] R. P. Stanley, Log-concave and unimodal sequences in algebra, combinatorics, and geometry, Graph Theory and Its Applications: East and West, Ann. New York, Acad. Sci. 576 (1989), 500-535.

[Wac 85] M. L. Wachs, Flagged Schur functions, Schubert polynomials, and symmetrizing operators, J. Combin. Theory Ser. A 40 (1985), 276-289.

Department of Mathematics, Michigan State University, East Lansing, Michigan 48824-1027 